\title{
High-Quality Experimental Data in Electron Microscopy and Microanalysis - What Can, and Should We Jointly Do?
}

\author{
Vasile-Dan Hodoroaba ${ }^{1}$ \\ 1. Federal Institute for Materials Research and Testing (BAM), Division 6.1 Surface Analysis and \\ Interfacial Chemistry, Berlin, Germany.
}

The basic principles of electron microscopies and microbeam analysis have been established many decades ago. The data provided are of continuously higher quality, this owing mainly to the technological developments in instrumentation (hardware and software) and computer performance. Further, the knowledge and skills of the operators must be also kept updated correspondingly. In order to be deemed as technical competent, the laboratories must be accredited according to an accreditation scheme, mostly following ISO/IEC 17025 "General requirements for the competence of testing and calibration laboratories" [1]. Thus, the quality system implemented aims at improving the laboratory's ability to consistently produce valid results.

There are different ways how to prove the quality of the analytical results obtained in a laboratory, e.g. use of validated standard operation procedures, participation in proficiency testing exercises, use of certified reference materials, etc. International standards provide requirements, specifications, guidelines or characteristics of methods, instruments or samples with the final goal that these can be used consistently in accredited laboratories. In the field of electron microscopy and microbeam analysis standardization and metrology are terms which are encountered rather seldom at major conferences and scientific publications. Nevertheless, spectra formats like EMSA/MSA for spectral-data exchange or tagged image file format (TIFF) for SEM, guidelines for performing quality assurance procedures or for the specification of X-ray spectrometers as well as of certified reference materials (CRMs) in EPMA, or measurement of average grain size by electron backscatter diffraction (EBSD), or guidelines for calibrating image magnification in SEM or TEM are ISO standards already published and used successfully by a large part of the electron microscopy and microbeam analysis community [2]. A main and continuous task of ISO/TC 202 and its subcommittees is to identify and evaluate feasible projects/proposals to be developed into new international standards, particularly with respect to recent but established technology, such the silicon drift detector (SDD) EDS.

As the metrological aspects are regarded, The International Bureau of Weights and Measures (BIPM) through the Consultative Committee for the Amount of Substance (CCQM) is concerned with the metrological aspect in Chemistry and Biology [3]. In particular, the Surface Analysis Working Group (SAWG) assists in identifying and establishing inter-laboratory work to test the consistency as well as to improve the traceability of spatially resolved chemical surface analysis at the micro and nanoscale. Examples of recent projects related to microbeam analysis, particularly focused on the quantification of light elements such as carbon and nitrogen, and the analytical challenges (analysis at low energies) associated with this task will be presented. The significant sources of measurement uncertainty will be emphasized through examples (see results at two different excitations in Fig. 1), i.e. quality of the specimen to be analyzed as well as of the reference materials selected, effect of the quantitation model, and the instrumental parameters like take-off-angle, spectrometer efficiency, and particularly the beam current. The crucial importance of working with uniform and well-defined measurement and data evaluation protocols will be discussed in compliance with the ISO document "Guide to the expression of 
uncertainty in measurement (GUM)" [5].

Another international platform in the frame of which pre-standardization work can be organized is VAMAS (Versailles Project on Advanced Materials and Standards) [6]. International collaborative projects involving aim at providing the technical basis for harmonized measurements, testing, specifications, and standards to be further developed at ISO level. One key point of VAMAS activities is constituted by inter-laboratory comparisons for high-quality data. In the field of microbeam analysis, the technical working area (TWA) 37 Quantitative Microstructural Analysis deals with corresponding projects. Good ideas, e.g. on analysis of low-Z materials/elements and at low energies are particularly encouraged by directly contacting the author. Support and already available guidance will be supplied.

References:

[1] ISO/IEC 17025 ISO, Geneve (2005).

[2] Technical Committees / ISO/TC 202 Microbeam analysis http://www.iso.org

[3] http://www.bipm.org/en/committees/cc/ccqm/.

[4] V-D Hodoroaba et al., Microsc. Microanal. 20 (S3) (2014), p. 730.

[5] ISO/IEC Guide 98-3:2008

(GUM:1995), Geneva: Internat. Org. Stds.

[6] Technical Working Area TWA 37 Quantitative Microstructural Analysis, http://www.vamas.org/
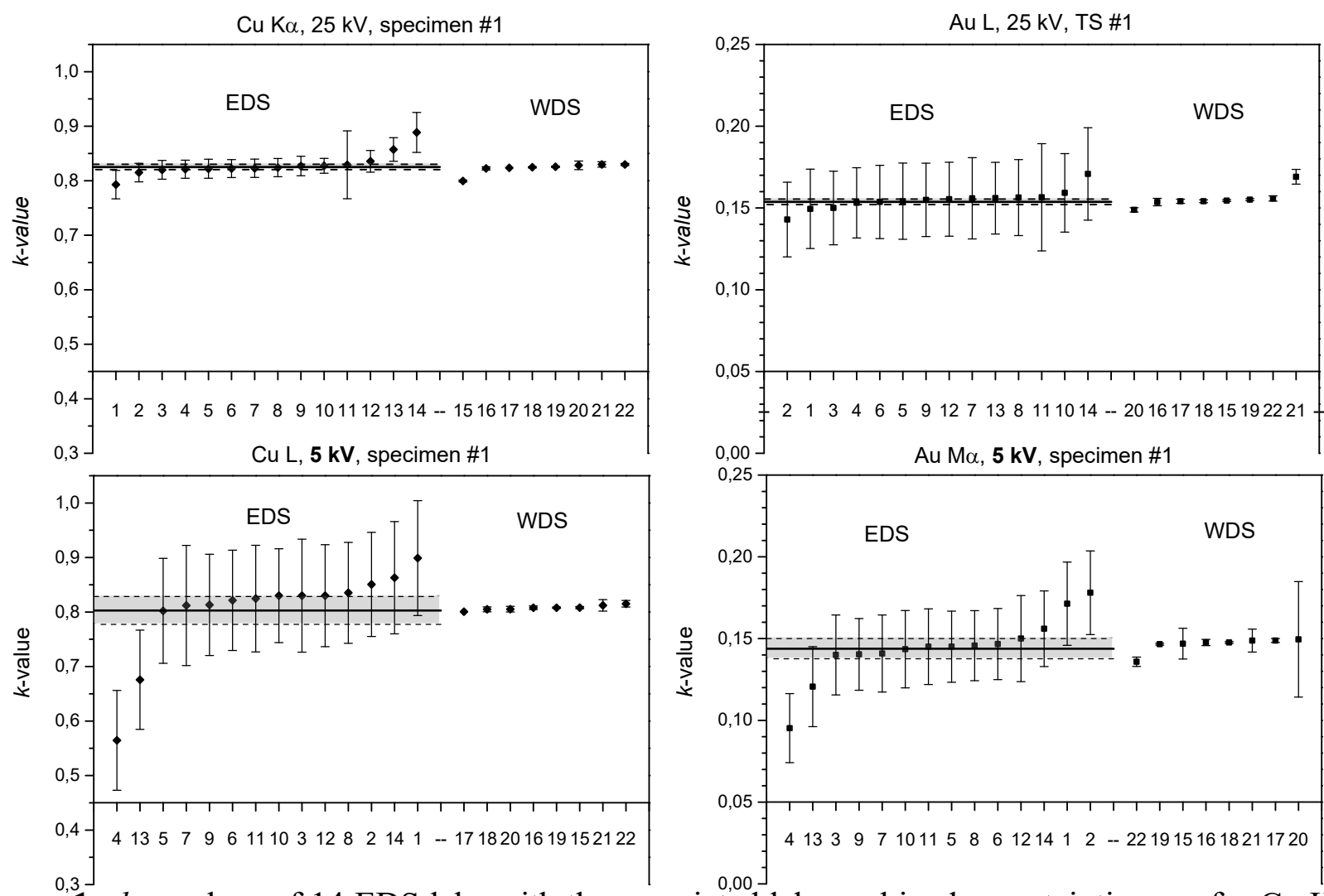

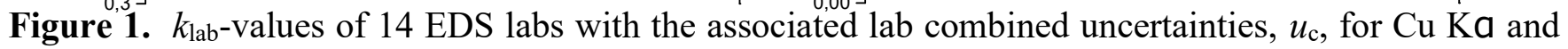
$\mathrm{Au} \mathrm{La}$ at $25 \mathrm{kV}$ (upper plots) and for $\mathrm{Cu} \mathrm{La}$ and $\mathrm{Au} \mathrm{Ma}$ at $5 \mathrm{kV}$ (lower plots) - for the test specimen \#1

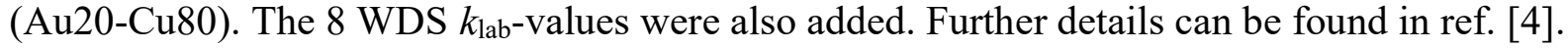

